\title{
BIOESTRATIGRAFÍA DEL JURÁSICO INFERIOR DE LA CUENCA DE LA RAMADA, ALTA CORDILLERA DE SAN JUAN (ARGENTINA)
}

\author{
P. Pamela ÁLVAREZ
}

Universidad de Buenos Aires - CONICET. Pabellón II, Departamento de Geología, Facultad de Ciencias Exactas y Naturales, Ciudad Universitaria (1428), Buenos Aires, Argentina.

Álvarez, P.P. 1996. Bioestratigrafía del Jurásico Inferior de la Cuenca de La Ramada, Alta Cordillera de San Juan (Argentina). [Lower Jurassic biostratigraphy of La Ramada Basin, High Cordillera of San Juan (Argentina)]. Revista Española de Paleontología, 11 (1), 35-47. ISSN 0213-6937.

\begin{abstract}
New biostratigraphic data are presented on the Jurassic marine sequences of La Ramada Basin, in the High Andes of San Juan province, Argentina. A number of different assemblage zones are recognised in the marine carbonate deposits of the Los Patillos Formation. These assemblage zones were known already for the Lower Jurassic of the Andean area, and they are correlated here with the standard European zones. Three new Lower Jurassic stratigraphic sections are described, and the first correlation is given between these and other sections in the La Ramada Basin.

The following Lower Jurassic biostratigraphic units are recognised: Fanninoceras Assemblage Zone, Radulonectites sosneadoensis Assemblage Zone, upper Pliensbachian; Posidonotis cancellata Assemblage Zone, Rhynchonelloidea cuyana Assemblage Zone, Dactylioceras hoelderi Assemblage Zone, Peronoceras largaense Assemblage Zone, Peronoceras pacificum Assemblage Zone, Collina chilensis Assemblage Zone, Phymatoceras toroense Assemblage Zone, Phlyseogramoceras tenuicostatum Assemblage Zone, Pleydellia lotharingica Zone and Pleydellia fluitans Zone, Toarcian.

The most characteristic components of the different assemblages are illustrated.
\end{abstract}

Keywords: Lower Jurassic, biostratigraphy, ammonites, Argentine Andes.

\section{RESUMEN}

Son presentados nuevos datos bioestratigráficos de las secuencias jurásicas marinas de los Andes de San Juan, Argentina. Los estudios bioestratigráficos realizados en las secuencias calcáreas marinas de la Formación Los Patillos han permitido reconocer diferentes zonas de asociación. Estas asociaciones han sido previamente establecidas en las cuencas Andinas y correlacionadas con las zonas estándar europeas. En este trabajo se presentan tres nuevos perfiles de las secuencias del Jurásico Inferior y la primera correlación entre estos y otras secciones de la Cuenca de La Ramada.

El Jurásico Inferior está representado por las siguientes unidades bioestratigráficas: Zona de Asociación de Fanninoceras, Zona de Asociación de Radulonectites sosneadoensis, Pliensbachiense superior; Zona de Asociación de Posidonotis cancellata, Zona de Asociación de Rhynchonelloidea cuyana, Zona de Asociación de Dactylioceras hoelderi, Zona de Asociación de Peronoceras largaense, Zona de Asociación de Peronoceras pacificum, Zona de Asociación de Collina chilensis, Zona de Asociación de Phymatoceras toroense Zona de Asociación de Phlyseogramoceras tenuicostatum, Zona de Asociación de Pleydellia lotharingica y Zona de Asociación de Pleydellia fluitans, Toarciense.

Diferentes láminas ilustran elementos distintivos de las diferentes asociaciones.

Palabras clave: Jurásico Inferior, bioestratigrafía, ammonites, Andes Argentinos.

\section{INTRODUCCIÓN}

La comarca de estudio se encuentra ubicada en la Cordillera Principal de los Andes entre los $31^{\circ} 40^{\prime} \mathrm{y}$ $32^{\circ} 20^{\prime}$ de latitud sur y los $70^{\circ} 30^{\prime}$ y los $70^{\circ} 10^{\prime} \mathrm{de}$ longitud oeste, en el extremo sudoccidental de la provincia de San Juan, Argentina (Fig. 1). En el ámbito de la Cuenca de la Ramada los perfiles estratigráficos levantados por la autora corresponden a la faja jurásica oriental que se encuentra entre los 69 $58^{\prime}$ y $70^{\circ} 09^{\prime}$ de longitud oeste y entre los $32^{\circ} 12^{\prime}$ y $32^{\circ} 18^{\prime}$ de latitud sur (Fig. 2).

Si bien los estudios paleontológicos realizados en el área se remontan a finales del siglo pasado, no se había 


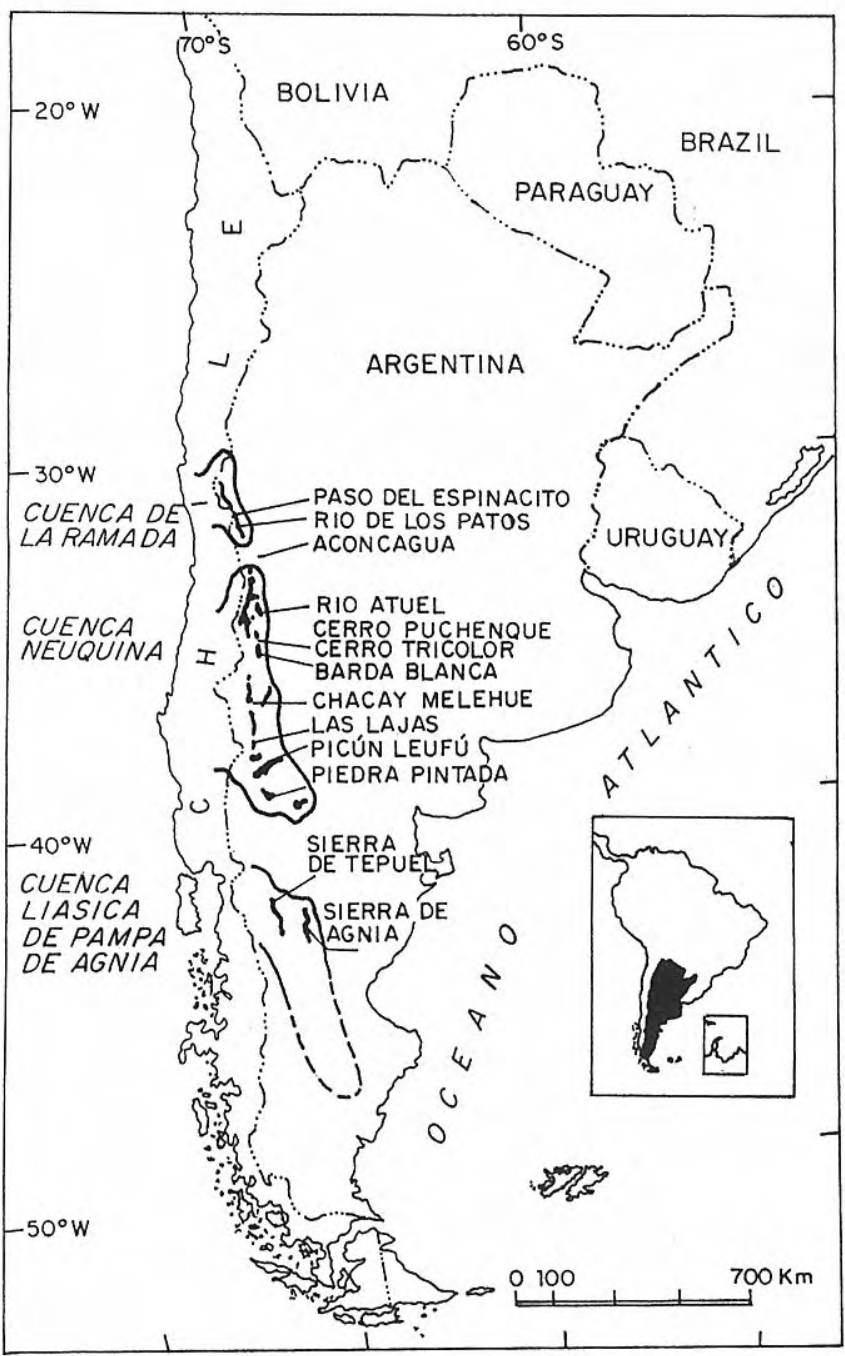

Figura 1. Cuencas liásicas Argentinas.

realizado aún un trabajo en donde se analizara pormenorizadamente el desarrollo del Jurásico Inferior marino en las diferentes fajas jurásicas de la Cuenca de la Ramada. Este trabajo tiene como objetivo principal presentar una correlación de perfiles, en donde se pueda observar la distribución geográfica de las distintas asociaciones fosilíferas con su exacta ubicación bioestragráfica.

Los primeros indicios de sedimentos jurásicos en la Alta Cordillera de San Juan fueron dados por Stelzner (1873), quien estudia estas secuencias en la clásica localidad del paso del Espinacito. Gottsche (1878) realiza el estudio paleontológico de la fauna de invertebrados coleccionada por Stelzner. Posteriormente Bodenbender, (en Törnquist, 1898) revisa nuevamente estas secuencias y reconoce las secciones del Jurásico Medio marino en el cordón de la Ramada. El material coleccionado fue descripto e ilustrado por Törnquist (1898).

Un estudio más regional del área fue llevado a cabo posteriormente por Schiller (1912) quien realiza perfiles estratigráficos y estructurales de estas secuencias a la latitud del paso del Espinacito (Figura 2).

Sin embargo, se debe a Rigal (1930) el hallazgo de Jurásico Inferior marino en la Alta Cordillera de San
Juan. El estudio de estas secuencias es ampliado posteriormente por Lambert (1943 y 1944) y Volkheimer et al. (1978 a y b) en el sector del arroyo de la Laguna.

Stipanicic (1966) describió las secuencias jurásicas en las localidades del paso del Espinacito, los Erizos, Yeguas Heladas y arroyo de las Flores, mencionando en este último la presencia de depósitos marinos del Toarciense.

Posteriormente, las faunas de invertebrados del Jurásico Inferior y Medio fueron estudiadas en distintas monografías (Westermann y Riccardi, 1972; Hillebrandt, 1987; Damborenea, 1987; Manceñido, 1991; Riccardi y Westermann, 1991; entre otros.)

Actualmente, el Área de Geotectónica de la Universidad de Buenos Aires está realizando el estudio de los afloramientos jurásicos en la Alta Cordillera Sanjuanina, cuyos primeros resultados han sido parcialmente dados a conocer por los trabajos de Álvarez (1991), Benoit (1992), Álvarez y Pérez (1993), Ramos et al. (1993), Álvarez et al. (1995) y Cristallini et al. (1995).

El material estudiado se encuentra depositado en las colecciones paleontológicas de la Universidad de Buenos Aires bajo las siglas CPBA.

\section{RESEÑA GEOLÓGICA}

Las unidades que afloran en el área de estudio comienzan con un basamento permotriásico correspondiente al Grupo Choiyoi, por encima del cual se encuentra en discordancia la Formación Rancho de Lata (Álvarez et al., 1995), del Triásico Superior-Jurásico Inferior, la cual se encuentra asociada a un volcanismo de tipo bimodal del Jurásico Inferior (Fig. 3).

En discordancia sobre estas unidades se desarrolla la Formación Los Patillos (Álvarez et al.,1995), cuya edad comprende el intervalo Pliensbachiense-Calloviense inferior. A su vez, sobre esta última unidad se superpone la Formación La Manga, litológicamente constituída por calizas mudstones y packestones, y brechas calcáreas. La secuencia marina culmina con las evaporitas de la Formación Auquilco, que están cubiertas por las areniscas rojas continentales de la Formación Tordillo. En discordancia angular sobre las unidades anteriormente mencionadas se encuentran los depósitos volcánicos y volcaniclásticos de la Formación Farellones, de edad miocena (Fig. 3).

\section{EL JURÁSICO INFERIOR MARINO EN LA CUENCA DE LA RAMADA}

En el ámbito de la Cordillera Principal Sanjuanina, Ramos et al. (1993) han identificado tres fajas de afloramientos jurásicos, una oriental, una central y una occidental. El presente trabajo se concentra principalmente en la faja oriental donde están mejor representadas las secuencias del Jurásico Inferior. Esta faja de afloramientos se desarrolla en la vertiente occidental del cordón del Espinacito desde las nacientes 


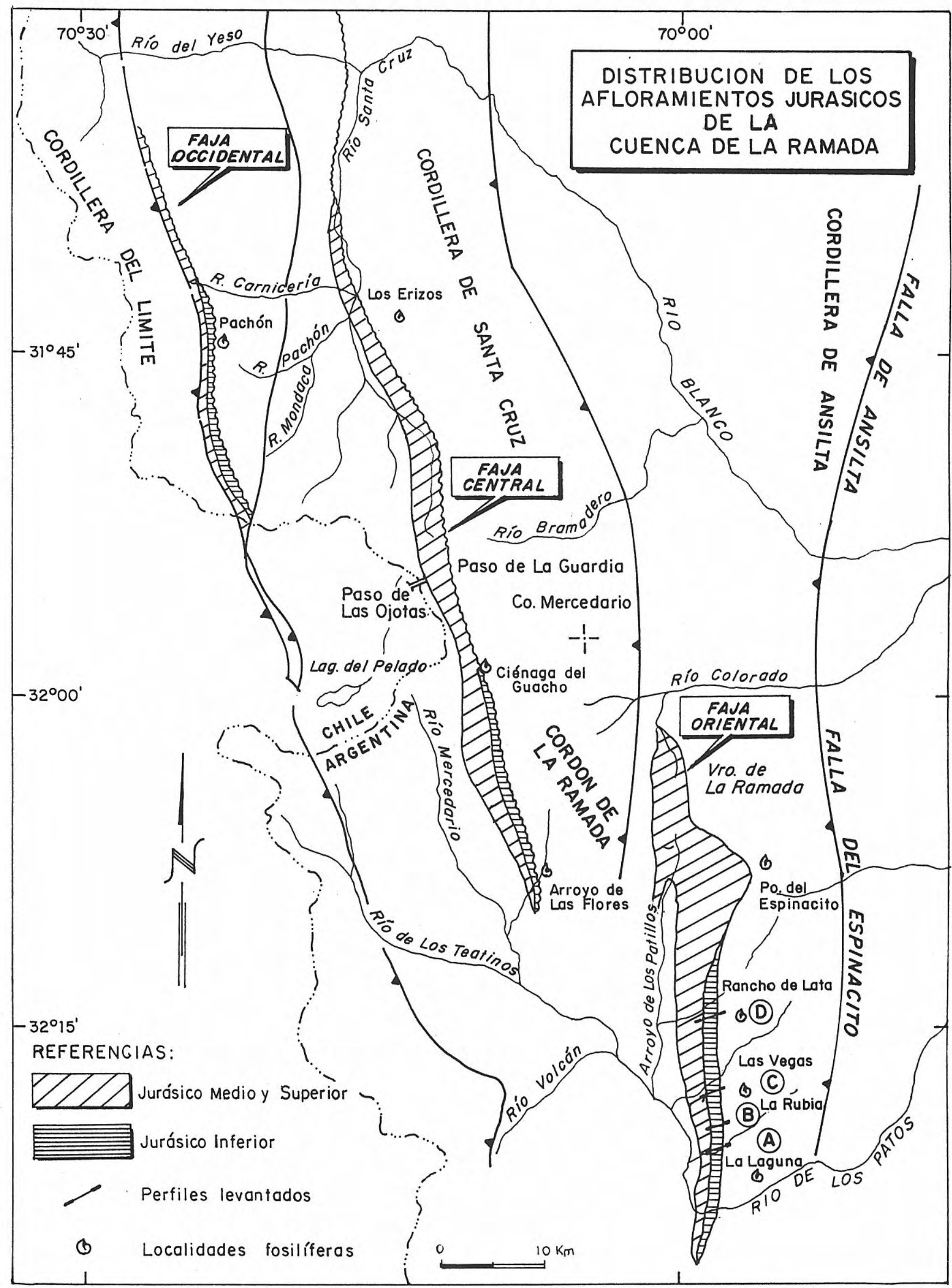

Figura 2. Distribución de los afloramientos liásicos de la Cuenca de la Ramada (modificado de Ramos et al., 1993). 


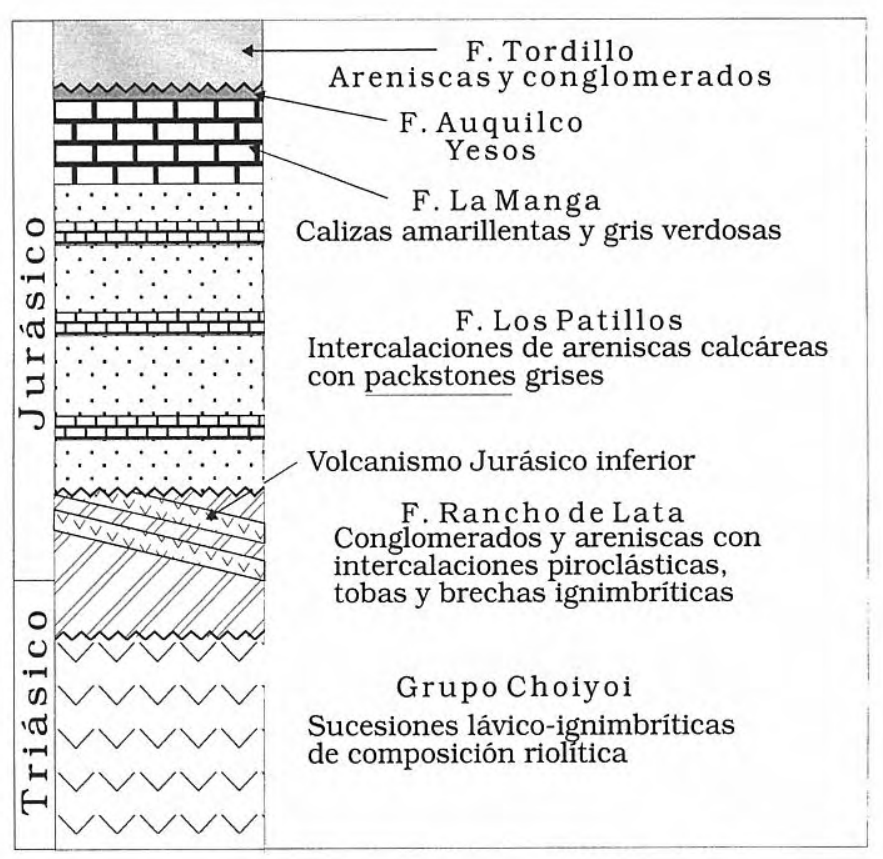

Figura 3. Esquema estratigráfico de las unidades jurásicas de la Cuenca de la Ramada.

del río Colorado en el sector norte, hasta la cordillera de los Penitentes al sur (Fig.2).

Las facies marinas del Jurásico Inferior están representadas en estas latitudes por los materiales basales de la Formación Los Patillos (Álvarez et al., 1995). Litológicamente están constituídos por rocas carbonáticas con intercalaciones de areniscas calcáreas, areniscas tobáceas y calizas bioclásticas (Álvarez et al., 1995). Generalmente se observan los niveles con mayor contenido paleontológico y en mejor estado de conservación en los bancos más calcáreos.

Estos afloramientos tienen su principal desarrollo en sentido norte-sur, acuñándose hacia el norte. Se puede observar una rápida disminución del espesor de los niveles pliensbachienses hacia el norte, los cuales prácticamente desaparecen a la latitud del arroyo Rancho de Lata (Fig. 4), en donde las secuencias marinas más antiguas con fauna de ammonites corresponden al Toarciense. Hacia el norte de esta localidad, los depósitos marinos toarcienses se interdigitan con secuencias continentales hasta desaparecer en el paso del Espinacito, donde los términos inferiores de la Formación Los Patillos son aalenienses (Hillebrandt, 1970; Álvarez, en prensa). De esta manera se documentan edades cada vez más recientes de la transgresión marina hacia el norte, en los afloramientos jurásicos de la faja oriental.

Dentro de la Cordillera Principal Sanjuanina se ha documentado la presencia de Jurásico Inferior tanto en la faja central como en la occidental, en donde los afloramientos se caracterizan litológicamente por tener niveles de areniscas blanquecinas intercaladas con pelitas grisáceas. En estas secuencias, los niveles arenosos presentan concentraciones locales de restos de invertebrados. Ramos et al. (1993) ilustran fósiles de estas secuencias de la faja occidental de afloramientos, en el cerro Los Fósiles, provincia de San Juan.

\section{BIOESTRATIGRAFÍA}

Los estudios bioestratigráficos realizados en la comarca han permitido reconocer una asociación muy completa de fósiles toarcienses. Dentro del área se conocen estudios previos realizados por Hillebrandt (1987), en la zona del arroyo Rancho de Lata (anteriormente denominado quebrada Honda) y por Volkheimer et al. (1978a), en la zona del arroyo de la Laguna, poniendo estos últimos autores especial énfasis en los niveles del Pliensbachiense y del Toarciense inferior. Estos niveles sólo están evidenciados por braquiópodos y bivalvos en los perfiles levantados. En este trabajo se presentan tres nuevos perfiles realizados en los arroyos de la Rubia, de las Vegas y Rancho de Lata, los que correlacionados con el perfil descripto por Volkheimer et al. (1978a), en el arroyo de la Laguna, permiten observar el desarrollo lateral de las secuencias del Jurásico Inferior (Fig. 4).

A continuación se describen las zonas bioestratigráficas reconocidas en la región estudiada, las cuales fueron definidas previamente en Argentina por Riccardi et al. (1990), y por Hillebrandt (1987), en el sector chileno.

\section{PLIENSBACHIENSE}

\section{Zona de Asociación de Fanninoceras}

Fue introducida por Riccardi (1984; véase también Riccardi et al. 1988; 1990). Los ammonites representativos de esta zona no han sido reconocidos en los arroyos de la Rubia, Rancho de Lata y de las Vegas. En el arroyo de la Laguna, Volkheimer et al. (1978a) determinan esta zona por la presencia de Protogrammoceras ex gr. normanianum (d'Orbigny) a 48,45 $\mathrm{m}$ de la base del perfil, documentando el Pliensbachiense superior.

En Argentina se ha ilustrado Fanninoceras oxyconum Hillebrandt, en la Formación Osta Arena de Pampa de Agnia (Chubut), (Blasco et al., 1979). En Mendoza, la Zona de Asociación de Fanninoceras se halla representada por una amplia franja que se extiende desde la región del río Atuel hasta el arroyo Chacay Co,

\section{Lámina I}

1-2 Weyla (Weyla) bodenbenderi (Behrendsen) (CPBA. 17528), vista anterior y vista lateral derecha.

3-4 Weyla (Weyla) alata angustecostata (Philippi) (CPBA 17457), vista lateral derecha y vista anterior.
5 Lobothyris subpunctata (Davidson) (CPBA 17450), vista dorsal.

6 Peristerothyris columbiniformis Manceñido (CPBA 17445), vista dorsal. 
Lámina I
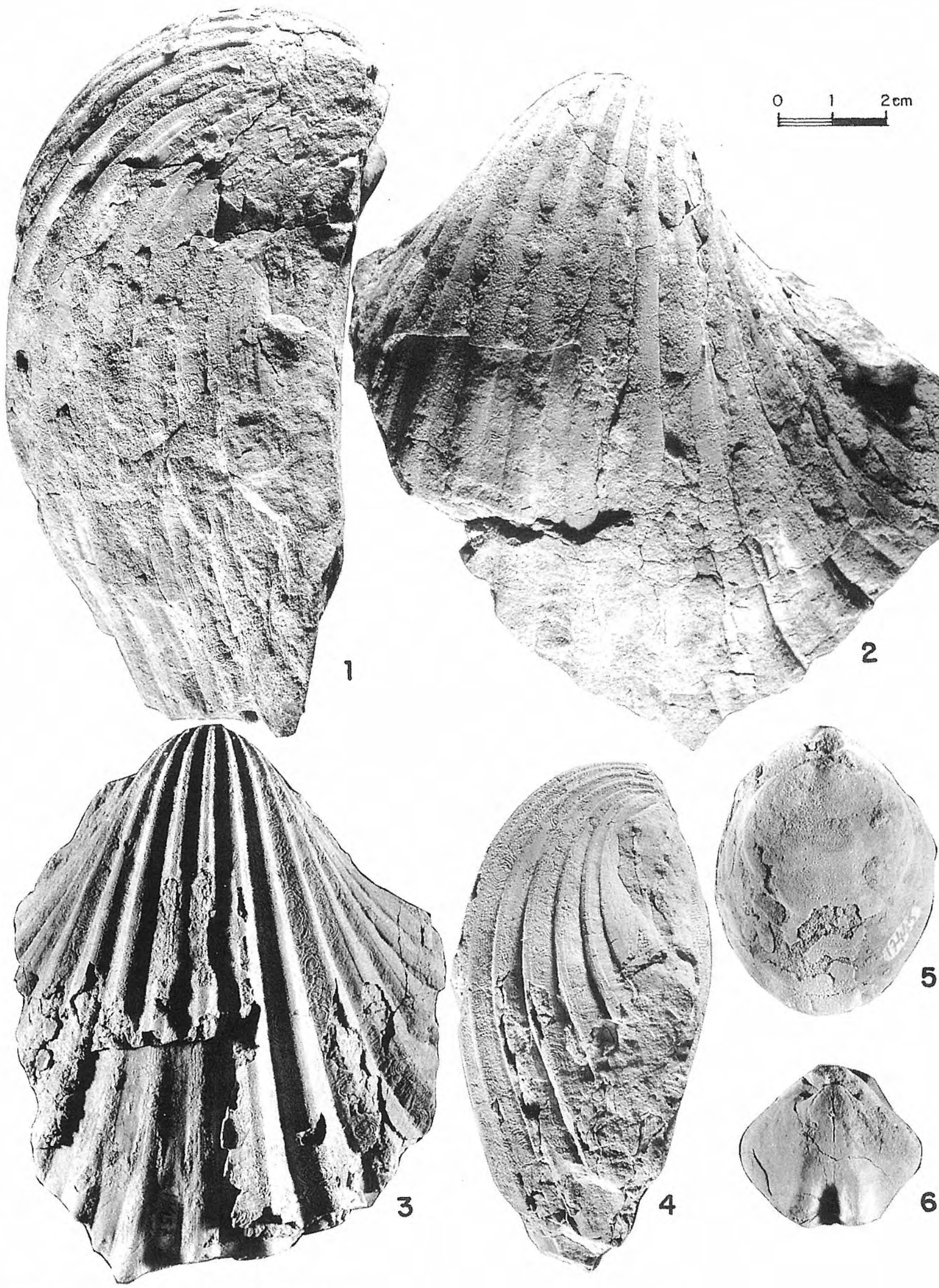

5

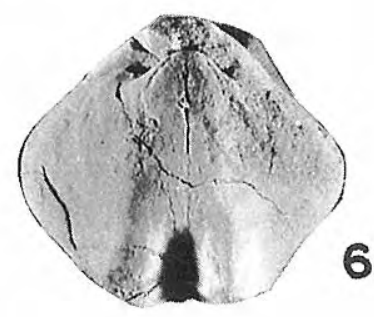

Revista Española de Paleontología, 11(1), 1996. 

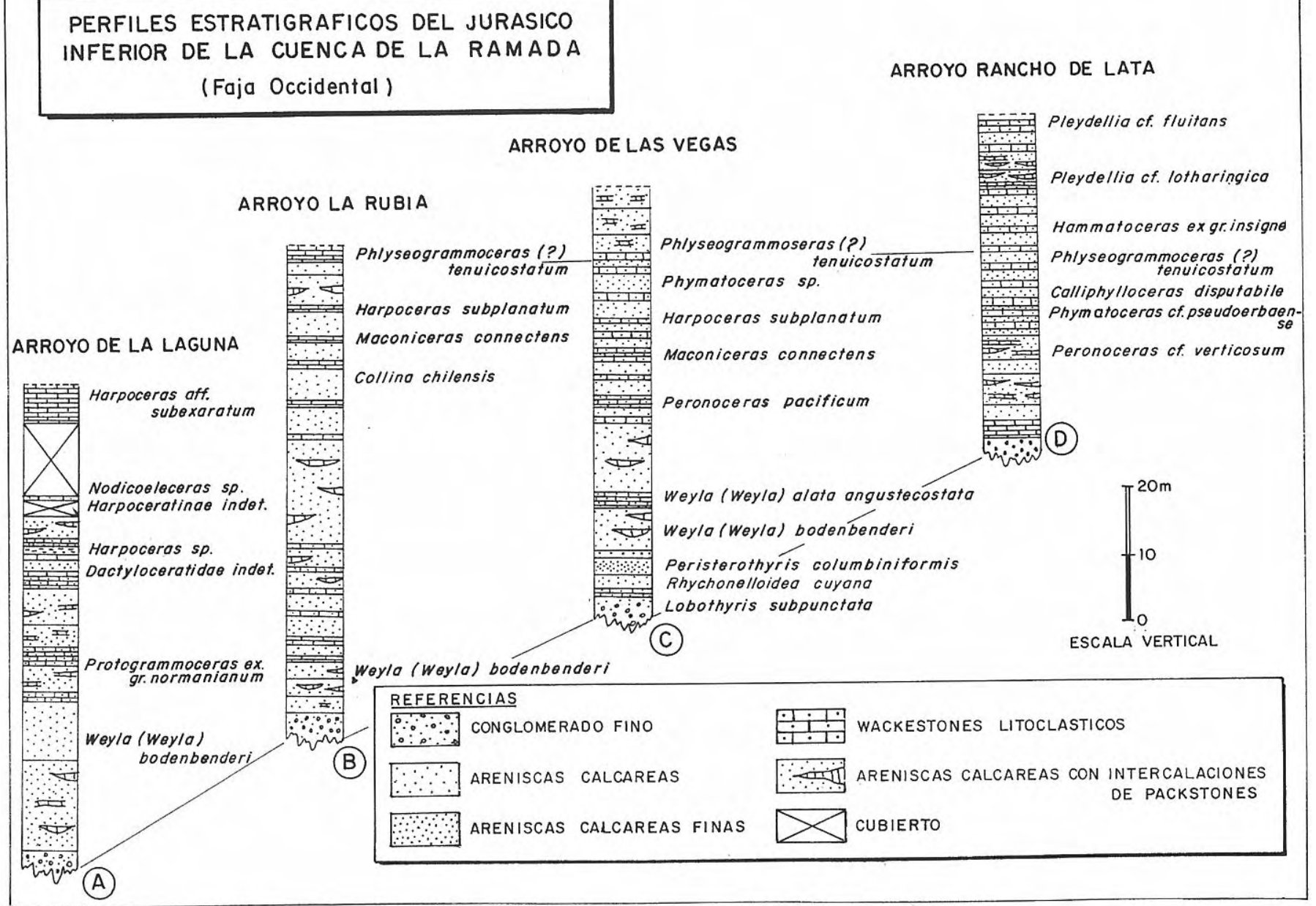

Figura 4. Perfiles estratigráficos del Jurásico Inferior de la Faja Occidental de afloramientos Jurásicos. (Perfil A, basado en Volkheimer et al. 1978 a y b).

inmediatamente al norte de Bardas Blancas. Esta zona está caracterizada por la presencia de Fanninoceras behrendseni (Jaworski), Protogrammoceras cf. normanianum (d'Orbigny), Arieticeras sp. y Fuciniceras sp. (Riccardi et al., 1994).

En el río Atuel, en el perfil de puesto Araya, Hillebrandt (1987) menciona la presencia de dos horizontes con Fanninoceras behrendseni (Jaworski).

Esta zona es parcialmente equivalente a las Zonas Estandar de Davoei, Margaritatus y Spinatum, de la zonación centro-europea (Riccardi et al., 1994) (Fig. 5).

\section{Zona de Asociación de Radulonectites sosneadoensis}

Esta asociación fue introducida por Damborenea (en Riccardi et al., 1990). Se reconoció en el arroyo de las Vegas con la presencia de Weyla (Weyla) bodenbenderi
(Behrendsen) a $20 \mathrm{~m}$ de la base del perfil. Al sur de esta localidad, en el arroyo de la Laguna, la presencia de esta especie se documentó a $47 \mathrm{~m}$ de la base del perfil (Volkheimer et al., 1978 a y b). En esta localidad se encontró junto con la siguiente fauna de bivalvos: Chlamys cf. prisca (Schlotheim), Myophorella cf. araucana (Leanza), Cardina sp., Pteromyia sp. y Gervilla sp.

En la localidad intermedia del arroyo de la Rubia se encontró Weyla (Weyla) bodenbenderi (Behrendsen) (Lám. I, figs. 1-2) a $25 \mathrm{~m}$ de la base del perfil (Figura 4).

En la Faja Central de la Alta Cordillera de San Juan, en la localidad del arroyo de las Flores, Ramos et al. (1993) mencionan la presencia de Weyla (Weyla) bodenbenderi (Beherensen) y Myophorella sp., aproximadamente a $25 \mathrm{~m}$ de la base del perfil, en bancos de lumaquelas intercalados entre pelitas gris-verdosas.

\section{Lámina II}

1 Harpoceras subplanatum (Oppel) (CPBA 17477), vista lateral.

2-3 Peronoceras pacificum Hillebrandt, (CPBA 17529), vista lateral y vista ventral.
4 Collina chilensis Hillebrandt (CPBA 17459), vista lateral.

5 Maconiceras connectens (Haug) (CPBA 17422), vista lateral. 
Lámina II
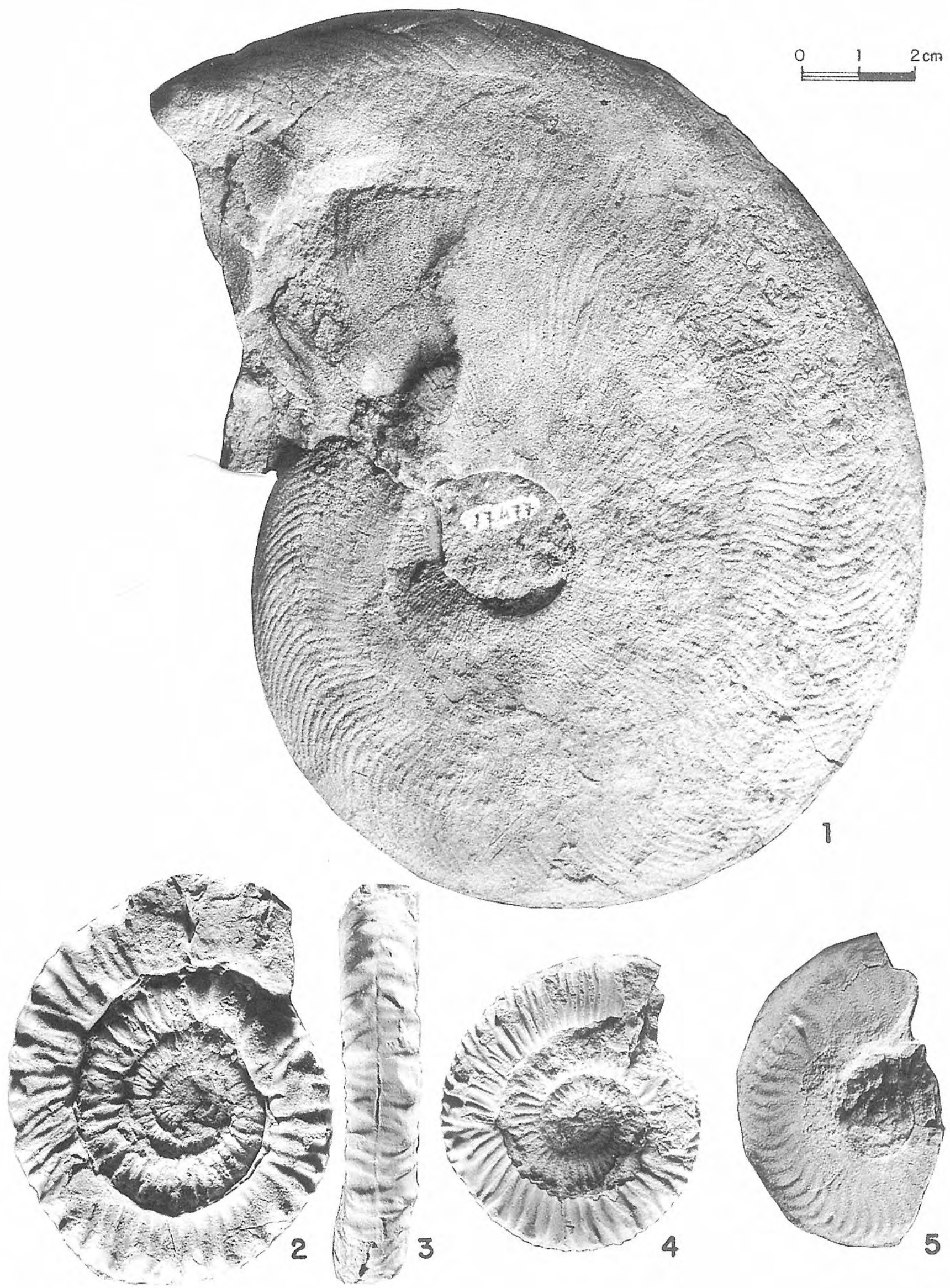

Rlevista Española de Paleontología, 11(1), 1996. 


\begin{tabular}{|c|c|c|c|c|c|c|}
\hline \multicolumn{2}{|c|}{$\mathrm{EDAD}$} & \multirow{4}{*}{$\begin{array}{l}\text { ZONAS ESTANDAR } \\
\text { DE AMMONITES } \\
\text { LEVESQUEI }\end{array}$} & \multicolumn{2}{|c|}{ ZONAS DE ASOCIACION DE AMMONITES } & $\begin{array}{l}\text { ASOCIACIONES } \\
\text { DE BIVALVOS }\end{array}$ & $\begin{array}{c}\text { ASOCIACIONES } \\
\text { DE BRAQUIOPODOS }\end{array}$ \\
\hline \multirow{11}{*}{ 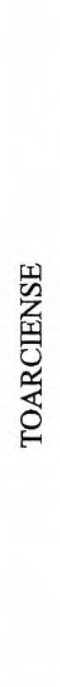 } & \multirow{5}{*}{ SUPERIOR } & & Z. "P. fluitans" & Fáunula Dumortieria & \multirow{9}{*}{$\begin{array}{l}\text { Propeamussium } \\
\text { cf. pumilus }\end{array}$} & \multirow{3}{*}{$\begin{array}{l}\text { Rhynchonelloidea } \\
\text { cf. ruthenensis }\end{array}$} \\
\hline & & & Z. "P. lotharingica" & \multirow{2}{*}{$\begin{array}{r}\text { Z. Phlyseogrammoceras } \\
\text { tenuicostatum }\end{array}$} & & \\
\hline & & & Z. P. tenuicostatum & & & \\
\hline & & THOUARSENSE & Z. P. copiapense & \multirow[b]{2}{*}{ Fáunula de Phymatoceras } & & \multirow[b]{2}{*}{$\begin{array}{l}\text { Prionorhynchia? } \\
\text { cf. rubrisaxensis }\end{array}$} \\
\hline & & VARLABILIS & Z. P. toroense & & & \\
\hline & \multirow{6}{*}{ INFERIOR } & \multirow{3}{*}{ BRIFRONS } & Z. C. chilensis & Z. Collina chilensis & & \multirow{4}{*}{ Rhynchonelloidea lamberti } \\
\hline & & & Z.P. pacificum & Z. Peronoceras pacificum & & \\
\hline & & & Z. P. largaense & Z. Peronoceras largaense & & \\
\hline & & FALCIFER & Z. D. hoelderi & Z. Dactylioceras hoelderi & & \\
\hline & & \multirow{2}{*}{ TENUICOSTATUM } & \multirow{2}{*}{ Z. D. tenuicostatum } & Z. D. tenuicostatum chilensis & \multirow{2}{*}{ Posidonotis cancellata } & \multirow{3}{*}{ Rhynchonelloidea cuyana } \\
\hline & & & & Z. Dactylioceras simplex & & \\
\hline \multirow{6}{*}{ 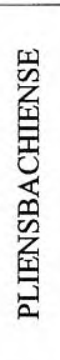 } & \multirow{2}{*}{ SUPERIOR } & SPINATUM & Z. F. disciforme & \multirow{3}{*}{ Z. Fanninoceras } & \multirow{2}{*}{$\begin{array}{l}\text { Radulonectites } \\
\text { sosnadoensis }\end{array}$} & \\
\hline & & MARGARITATUS & Z. F. fannini & & & \multirow{5}{*}{$\begin{array}{l}\text { Rhynchonelloidea } \\
\text { burckhardti }\end{array}$} \\
\hline & \multirow{4}{*}{ INFERIOR } & DAVOEI & Z. F. behrendseni & & \multirow{4}{*}{ Otapiria neuquensis } & \\
\hline & & IBEX & Z. E. meridianus & & & \\
\hline & & \multirow[b]{2}{*}{ JAMESONI } & Z."Tropidiceras" & Z. Dubariceras & & \\
\hline & & & Z. "Apoderoceras" & & & \\
\hline
\end{tabular}

Figura 5. Cuadro de correlación de unidades bioestratigráficas del Jurásico Inferior. Basado en Riccardi et al. (1990) y Manceñido (1990).

Weyla (Weyla) bodenbenderi es muy abundante en los depósitos litorales del Pliensbachiense superior de Argentina (Damborenea y Maceñido, 1988). Se ha mencionado la presencia de fauna característica de esta asociación en los perfiles de las localidades del paso de Portezuelo Ancho y Piedra Pintada, en Mendoza; río de los Patos, en San Juan y en las provincias de Neuquén y Chubut.

Esta asociación comprende una extensión similar a la Zona de Asociación de Fanninoceras (Fig. 5).

\section{TOARCIENSE}

\section{Zona de Asociación de Posidonotis cancellata}

Fue introducida por Damborenea (en Riccardi et al., 1990). En el arroyo de las Vegas está caracterizada por la presencia de Weyla (Weyla) alata angustecostata
(Philippi) (Lám.I, figs. 3-4), Gervilla sp. y Myophorella sp., a $22 \mathrm{~m}$ de la base del perfil (Fig. 2), documentando el Toarciense Inferior. La fauna perteneciente a esta asociación presenta una gran distribución geográfica en Argentina y ha sido documentada en las siguientes localidades: cordón del Espinacito, cerro Puchenque, cerro Tricolor, Chacay Melehue, Picún Leufú, cerro Lotena y Pampa de Agnia (véase Damborenea, en Riccardi et al. 1990).

Esta Zona de Asociación se corresponde con la parte superior de la Zona Estándar Spinatum y Tenuicostatum (Fig. 5).

\section{Zona de Asociación de Rhynchonelloidea cuyana}

Fue introducida por Manceñido (1991). En la región de estudio se encuentra representada por Rhynchonelloidea cuyana Manceñido, Peristerothyris columbini-

\section{Lámina III}

1-2 Phymatoceras cf. pseuderbaense (Gabilly) (CPBA $16514)$, vista ventral y lateral.

3 Hammatoceras ex gr. insigne (Zieten) (CPBA 16520), vista lateral.

4 Phlyseogrammoceras tenuicostatum (Jaworski) (CPBA 17402), vista lateral.
5 Calliphylloceras disputabile (Zittel) (CPBA 16507), vista lateral.

6-7 Pleydellia cf. fluitans (Dumortier) (CPBA 16505), vista ventral y lateral.

8 Pleydellia cf. lotharingica (Branco) (CPBA 16509), vista lateral. 
Lámina III

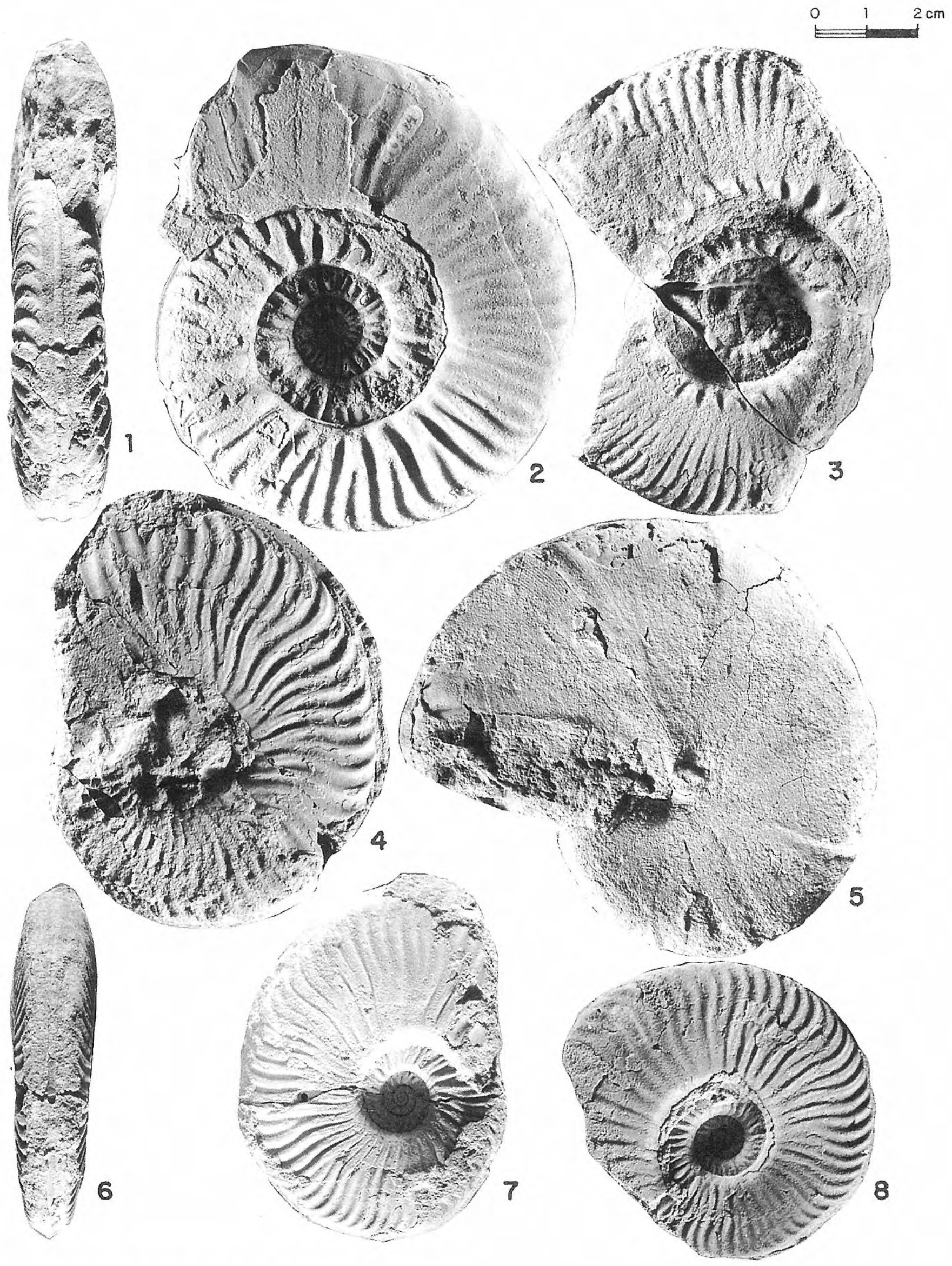

Revista Española de Paleontología, 11(1), 1996. 
formis Manceñido (Lám.1, fig. 6) y Lobothyris subpunctata (Davidson) (Lám. I, fig. 5). Esta asociación fue encontrada a $22 \mathrm{~m}$ de la base del perfil del arroyo de las Vegas.

En el arroyo de la Laguna, Volkheimer et al. (1978 a y b) mencionan la presencia de los géneros Lobothyris y Homoeorhynchia y de la especie Spiriferina aff. tumida (V. Buch).

En el arroyo de las Flores se ha reconocido la siguiente asociación: Rhynchonella cuyana Manceñido, Lobothyris subpunctata (Sowerby), Fissirhynchia piremapuana Manceñido y Peristerothyris columbiniformis Manceñido. La asociación se encontraba en lumaquelas intercaladas en pelitas gris-verdosas de $15 \mathrm{~m}$ de espesor (Ramos et al., 1993).

Esta zona abarca una extensión similar a la Zona Estándar Spinatum y Tenuicostatum (Fig. 5).

\section{Zona de Asociación de Dactylioceras hoelderi}

Fue introducida para Chile por Hillebrandt y SchmidtEffing (1981). En el cordón del Espinacito su presencia fue reconocida por Volkheimer et al. (1978 a y b), en el arroyo de la Laguna. Estos autores mencionan la presencia de Dactiloceratidae indet. y Harpoceras sp., a $72 \mathrm{~m}$ de la base del perfil; Nodicoeloceras sp. y Harpoceratinae indet., a $88 \mathrm{~m}$ de la base del perfil, indicativos del Toarciense inferior. Hillebrandt (1987), reconoce esta zona en Argentina en el arroyo Serrucho, por la presencia de Harpoceratoides cf. alternatus (Simpson) y Nodicoloceras cf. crassoides (Simpson). Existen numerosas evidencias de la presencia de fauna perteneciente a esta zona a la latitud de Malargüe (Mendoza) en la Formación Puchenque, hasta Catán Lil (Neuquén) en la Formación Los Molles (Riccardi, 1984) y en la Formación Osta Arena de Pampa de Agnia (Chubut) (Blasco et al., 1979).

Esta zona es parcialmente equivalente a la Zona de Estándar Falcifer de la zonación centroeuropea.

\section{Zonas de Asociación de Peronoceras largaense, $P$. pacificum y Collina chilensis}

Estas zonas fueron definidas por Hillebrandt (1987) para Chile y no se encuentran aún bien diferenciadas en la Argentina (Riccardi et al., 1990).

En el área del cordón del Espinacito se encuentran representadas en el perfil realizado en la quebrada del arroyo Rancho de Lata, por la presencia de Peronoceras verticosum (Buckman) a 40 metros de la base del mismo. Al sur de esta localidad, en el arroyo de las Vegas (Fig. 2), está caracterizada por Peronoceras pacificum Hillebrandt (Lám. II, fig. 4), Maconiceras connectens (Haug) y Harpoceras subplanatum (Oppel) (Lám. II, fig. 1) a 60 m de la base el primero y a $70 \mathrm{~m}$ de la misma el segundo.

En los últimos niveles del perfil del arroyo de la Rubia (Fig. 4), se observó la presencia de Collina chilensis Hillebrandt (Lám. II, figs. 2-3), por encima un nivel con Maconiceras connectes y otro con Harpoceras subplanatum, todos representativos de esta zona.
Dentro de la Cordillera Sanjuanina se ha documentado esta asociación en la localidad del arroyo de las Flores (Ramos et al., 1993), en donde se describe un nivel con Harpoceras subplanatum en concreciones arenosas aproximadamente a $45 \mathrm{~m}$ de la base del perfil.

En la Faja Jurásica Oriental, en la localidad del Cerro de los Fósiles, se han determinado dos niveles: uno inferior con Harpoceras subplanatum y Peronoceras sp. y otro superior, al sur de la primera localidad, con Harpoceras subplanatum y Maconiceras connectens (Ramos et al., 1993).

Han sido registradas en Mendoza en la Formación Puchenque del cerro Puchenque (Westermann y Riccardi, 1972; Hillebrandt, 1987, Riccardi et al., 1994). En el ámbito de la Cuenca Neuquina esta asociación se encuentra presente en la Formación Los Molles, en el área de Chacay Melehue, arroyo Lapa, arroyo La Jardinera, Catán Lil (Riccardi, 1984); y en la Formación Osta Arena, en la localidad de Pampa de Agnia (Chubut) (Riccardi, 1984).

En conjunto las tres zonas son aproximadamente equivalentes a la Zona Estándar Bifrons de la zonación centroeuropea (Fig. 5).

\section{Zona de Asociación de Phymatoceras toroense}

Fue reconocida por Hillebrandt (1987) en diferentes secciones del norte de Chile y para algunas localidades argentinas. En la comarca de estudio se encuentra representada, en el arroyo Rancho de Lata, por dos niveles con Phymatoceras cf. pseudoerbaense (Gabilly) (Lám. III, fig. 1-2) y Calliphylloceras disputabile (Zittel) (Lám. II, fig. 5), los cuales se encuentran aproximadamente a 34 $m$ de la base del perfil, y por Phymatoceras sp., en el arroyo de las Vegas, a $85 \mathrm{~m}$ de la base del mismo.

Esta zona es equivalente a la parte alta de la Zona de Brifons y a las Zonas de Variabilis y Thouarsense de la zonación europea (Hillebrandt, 1987).

Como puntualiza Riccardi (1984), en Argentina no se ha realizado una diferenciación estratigráfica como en Chile, por lo que la Zona de Phymatoceras toroense no ha sido individualizada. La misma se conoce como Fáunula de Phymatoceras y ha sido documentada en la Formación Bardas Blancas en Mendoza y en la Formación Los Molles en Neuquén.

\section{Zona de Asociación de Phlyseogrammoceras tenuicostatum}

Fue definida por Riccardi (1984; véase también Riccardi et al., 1990). Esta zona está caracterizada en el perfil del arroyo Rancho de Lata por dos niveles de Phlyseogrammoceras cf. tenuicostatum (Jaworski) (Lám. III, fig. 4 ) a $48 \mathrm{~m}$ de la base y por un nivel superior a los anteriores con Hammatoceras ex gr. insigne (Zieten) (Lám. III, fig. 3). Tanto en el arroyo de las Vegas, como en el arroyo de la Rubia se encuentra representada por Phlyseogrammoceras cf. tenuicostatum (Fig. 4). Esta zona es equivalente a la parte inferior de la Zona Estándar Levesquei de la zonación europea. 
Dentro de la cuenca de la Ramada, en la localidad de Ciénaga del Gaucho, Ragona (1993) menciona la presencia de Phlyseogrammoceras cf. tenuicostatum, aproximadamente a 100 metros de la base de los perfiles realizados en la quebrada de los Naipes y en la quebrada del Abanico.

En Argentina ha sido reconocida en la región de Malargüe en el arroyo Negro y arroyo de las Overas, en donde se ha encontrado esta especie junto a Witchellia obscurecostata (Jaworski) (Hillebrandt, 1987). En el área de estudio fue reconocida asociada a Hammatoceras ex gr. insigne (Schubler) y Sphaerocoeloceras brochiiforme (Jaworski) (Hillebrandt, 1987).

Riccardi (1984) menciona la presencia de fauna correspondiente a esta zona en la Formación Tres Esquinas (=China Muerta) del arroyo Blanco, cerro Tricolor, arroyo Chacaico, cerro Puchenque (Mendoza), y en la Formación Los Molles del arroyo Niraico, Picún Leufú y arroyo Lapa (Neuquén).

\section{Zona de "Pleydellia lotharingica"}

Fue introducida por Hillebrandt (1987). En el perfil realizado en la ladera sur del arroyo Rancho de Lata se han identificado dos niveles con Pleydellia cf. lotharingica (Branco) (Lám. III, fig. 8), encontrándose a $62 \mathrm{~m}$ y a $65 \mathrm{~m}$, respectivamente, de la base del perfil. Es equivalente a la parte superior de la Zona Estándar Levesquei en la zonación europea.

En la Faja Central de afloramientos jurásicos, Ragona (1993) describe la presencia de Pleydellia cf. lotharingica en el perfil realizado en la quebrada de los Naipes, en la localidad de Ciénaga del Gaucho (Fig. 2)

En Argentina, esta zona de asociación se conoce como Fáunula Dumortieria (Fig. 5), ya que no se ha realizado una diferenciación estratigráfica como en el sector chileno. Fauna perteneciente a la misma ha sido hallada en la Formación Los Molles de Chacay Melehue, arroyo Tocuyo, cerro Lapa y Chacaico en la provincia de Neuquén (Riccardi et al., 1993).

\section{Zona de "Pleydellia fluitans"}

Fue introducida por Hillebrandt (1987). Esta zona se encuentra representada en el perfil del arroyo Rancho de Lata por la presencia de dos niveles con Pleydellia cf. fluitans (Dumortier) (Lám. III, figs. 6-7), que se encuentran aproximadamente a $5 \mathrm{~m}$ del último de los bancos con Pleydellia cf. lotharingica (Dumortier). Esta zona ya había sido reconocida por Hillebrandt (1987) en la comarca de estudio para el perfil de la quebrada Honda, actualmente denominada Rancho de Lata.

En Argentina, los representantes de esta zona se han encontrado en las mismas localidades en que se reconoció la presencia de la Zona de Pleydellia cf. lotharingica (Riccardi et al, 1993).

\section{CONCLUSIONES}

El estudio realizado ha permitido llegar a las siguientes resultados:
1. Se presentan los primeros perfiles estratigráficos del Toarciense marino de la Alta Cordillera de San Juan.

2. Con base en las asociaciones fósiles se puede documentar la edad de la transgresión marina en la faja oriental de afloramientos jurásicos. La misma se inició durante el Plienbaschiense tardío, correspondiéndose con uno de los pulsos transgresivos importantes señalado por Hallam (1991) en los cambios globales del nivel del mar para los Andes.

A diferencia de la cuenca Neuquina y otras cuencas jurásicas del sector chileno, en donde la transgresión marina comenzó en el Triásico Superior - Jurásico Inferior temprano (Legarreta et al., 1993), la cuenca de la Ramada durante el Hettangiense - Sinemuriense se comporta como un alto topográfico que no permite la trangresión del mar sino hasta el Pliensbachiense medio tardío.

3. Se realiza la primera correlación bioestratigráfica de perfiles, lo que permite observar el desarrollo lateral de las secuencias del Jurásico Inferior. Los datos bioestratigráficos indican un rápido acuñamiento hacia el sector norte de los niveles marinos pliensbachienses, que desaparecen inmediatamente al norte de la localidad del arroyo Rancho de Lata. Este comportamiento de las secuencias pliensbachienses corresponde a un control paleogeográfico, con un paleorelieve originado por un sistema de bloques fallados formando grabens y semigrabens, característico de las cuencas de rift. Este paisaje fue parcialmente suavizado, durante el Triásico Medio - Superior, por el desarrollo de los depósitos correspondientes a la Formación Rancho de Lata, los cuales, sin embargo, no pudieron obliterar el marcado control del paleorelieve (Ramos y Álvarez, en prensa). Con base en los estudios realizados en el área, el cambio en el desarrollo de las secuencias jurásicas de esta edad, exactamente al norte del arroyo Rancho de Lata, se interpreta como correspondiente al borde de uno de estos bloques, marcando un relieve progresivamente más alto hacia el paso del Espinacito. Un desarrollo similar se observa en las secuencias de edad toarciense.

\section{AGRADECIMIENTOS}

Se desea agradecer a V. Ramos y a M. B. Aguirre Urreta (Universidad de Buenos Aires) por la lectura del texto y las importantes sugerencias realizadas. A A.C. Riccardi, M. Manceñido y S. Damborenea (Museo de la Plata) por la revisión de la clasificación de los ammonites, braquiópodos y bivalvos mencionados en el texto. A S. Benoit, D. Perez y B. Aguirre Urreta que participaron en las tareas de campaña en 1990 y a D. Charchaflié que colaboró en las tareas de campo en 1993.

Este trabajo ha sido parcialmente financiado con los siguientes subsidios: UBACYT Ex 132 y PID CONICET 330830092 .

Esta es una contribución al proyecto IGCP 322 "Correlación de Eventos Jurásicos en Sudamérica". 


\section{BIBLIOGRAFÍA}

Álvarez, P.P. 1991. Estudio geológico del arroyo Rancho de Lata, Cordón del Espinacito. Universidad de Buenos Aires, Trabajo final de Licenciatura, 182 págs., Buenos Aires (inédito).

Álvarez, P. P. (en prensa). Jurassic ammonite assemblages and zones of the Principal Cordillera of San Juan, Argentina. IV International Congress on Jurassic Stratigraphy and Geology, Mendoza.

Álvarez, P.P. y Pérez, D.J. 1993. Estratigrafía y estructura de las nacientes del río Colorado, Alta Cordillera de San Juan. XII Congreso Geológico Argentino y II Congreso de Exploración de Hidrocarburos, Actas, 2, 78-84.

Álvarez, P.P., Benoit, S.V. y Ottone, E.G.1995. Las formaciones Rancho de Lata y Los Patillos en el Mesozoico de la Cordillera Principal de San Juan. Revista de la Asociación Geológica Argentina, 49 (1-2), (1994), 133-152.

Benoit, S.V. 1992. Estudio geológico de los depósitos triásico-jurásicos del extremo sur de la vertiente occidental del cordón del Espinacito (Provincia de San Juan). Trabajo Final de Licenciatura, Universidad de Buenos Aires, 110 págs., Buenos Aires (inédito).

Blasco, G., Levy, R. y Nullo, F. 1979. Los amonites de la Formación Osta Arena (Liásico) y su posición estratigráfica. Pampa de Agnia (Provincia de Chubut). VII Congreso Geológico Argentino, Actas, 2, 407-429.

Cristallini, E.O., Mosquera A. y Ramos, V.A. 1995. Estructura de la Alta Cordillera de San Juan. Revista de la Asociación Geológica Argentina, 49 (1-2), (1994), 165-183.

Damborenea, S.E. 1987. Early Jurassic bivalvia of Argentina. Part 1: Stratigraphical introduction and superfamilies Nuculanacea, Arcacea, Mytilacea and Pinnacea. Palaeontographica (A), 199 (1-3), 1-111.

Damborenea S.E. y Maceñido M.O. 1988. Weyla: Semblanza de un bivalvo jurásico andino. $V$ Congreso Geológico Chileno, Actas, 2, C13-C25.

Gottsche, C. 1878. Uber Jurassische Versteinerungen aus der Argentinischen Cordillere. Palaeontographica, Suplement III, 2(3), 1-50.

Hallam, A. 1991. Relative importance of regional tectonics and eustasy for the Mesozoic of the Andes. In: Sedimentation, Tectonics and Eustasy (Ed. D.I.M. McDonald). Special Publication int Association Sedimentology, 12, 189-200.

Hillebrandt, A. von 1970. Zur Biostratigraphie und Ammoniten-Fauna des südamerikanischen Jura (insbes. Chile). Neues Jahrbuch für Geologie und Pälaontologie, Abhandlungen 136, 166-211.

Hillebrandt, A. von 1987. Liassic ammonite zones of South America and correlations with other province. With description of new genera and species of ammonites. En Bioestratigrafía de los Sistemas regionales del Jurásico y Cretácico de America del Sur (Ed. W. Volkheimer) I, 111-157.

Hillebrandt, A. von und Schmidt-Effing, R. 1981. Ammoniten aus dem Toarcium (Jura) von Chile (Südamerika). Die Arten der gattungen Dactylioceras,
Nodicoeloceras, Peronoceras und Collina. Zitteliana, 6 , $1-74$.

Lambert, L. 1943. Perfil geológico en el valle superior del río de Los Patos Sur (Provincia de San Juan). Revista del Museo de Ciencias Naturales de La Plata (n.s.) Geología, 2 (11), 1-10.

Lambert, L. 1944. Informe sobre un proyecto de dique de embalse del Río de los Patos sur. Boletín de la Dirección de Mineralogía, Geología e Hidrogeología de la Nación, $57,1-48$.

Legarreta, L., Gulisano, C.A. y Uliana, M.A. 1993. Las secuencias sedimentarias jurásico-cretácicas. XII Congreso Geológico Argentino y II Congreso de Exploración de Hidrocarburos (Mendoza, 1993), Geología y Recursos Naturales de Mendoza, Relatorio (Ed. V.A. Ramos), 2 (9), 87-114.

Manceñido, M.O. 1991. The succesión of Early Jurassic braquiopod faunas from Argentina: correlations and affinities.In Brachiopods through Time, Proceedings of the 2nd International Brachiopod Congress (Eds. D.I. MacKinnon, D.E. Lee and J.D. Campell) Balkema, Rotterdam, 397 - 404.

Ragona, D. 1993. Estudio geológico de la Cienaga del Gaucho, Alta Cordillera de San Juan. Universidad de Buenos Aires, Trabajo final de Licenciatura, 113 p., Buenos Aires (inédito).

Ramos, V.A. and Álvarez, P.P. (en prensa). Early Mesozoic tectonics of the High Andes of Argentina - Chile. IV International Congress on Jurassic Stratigraphy and Geology, Mendoza.

Ramos, V.A., Aguirre Urreta, M.B. y Lencinas, A. 1993. El Toarciano fosilífero de Pachón y sus relaciones con el Jurásico marino de la Cordillera Principal de San Juan. XII Congreso Geológico Argentino y II Congreso de Exploración de Hidrocarburos, Actas, 2, 94-103.

Riccardi, A.C., 1984. Las asociaciones de amonitas del Jurásico y Cretácico de la Argentina. IX Congreso Geológico Argentino, Actas, 4, 559-595.

Riccardi, A.C. and Westermann, G.E.G. 1991. Middle Jurassic Ammonoid Fauna and Biochronology of the Argentine-Chilean Andes. Part 3: Bajocian-Callovian Eurycephalitinae, Stephanocerataceae. Palaeontographica A, 216, 1-110.

Riccardi, A.C., Damborenea, S.E. y Manceñido, M.O. 1988. Hettangiano y Sinemuriano marinos en Argentina. $V^{\circ}$ Congreso Geológico Chileno, Actas, II(C), 359-373.

Riccardi, A.C., Damborenea, S.E. and Manceñido, M.O. 1990. Lower Jurassic of South America and Antarctic Peninsula. In Jurassic taxa ranges and correlations charts for the Circum - Pacific (Eds. G. E. G. Westerman and A. C. Riccardi). Newsletters on Stratigraphy, 21 (2), 129-147.

Riccardi, A.C., Damborenea, S.E. y Manceñido, M.O. 1993. Invertebrados mesozoicos. XII Congreso Geológico Argentino y II Congreso de Exploración de Hidrocarburos (Mendoza, 1993), Geología y Recursos Naturales de Mendoza, Relatorio (Ed. V. A. Ramos), 2 (12), 329 - 340.

Rigal, R., 1930. El Liásico en la Cordillera del Espinacito (Provincia de San Juan). Publicación de la Dirección Nacional Geología y Minería, 74, 5-93. 
Schiller, W. 1912. La Alta Cordillera de San Juan y Mendoza y parte de la provincia de San Juan. Anales del Ministerio de Agricultura, Sección Geología, Mineralogía y Minería, 7 (5), 1-68.

Stelzner, A., 1873. Uber die argentinische Cordillera zw. $31^{\circ}$ und $33^{\circ}$ S. Neues Jahrbuch für Mineralogie, Geologie und Pälaontologie, 8, 726-744.

Stipanicic, P. N. 1966. El Jurásico en Vega de La Veranada (Neuquén), el Oxfordense y el diastrofismo Divesiano (Agassiz-Yaila) en Argentina. Revista de la Asociación Geológica Argentina, 20, 403-478.

Törnquist, A. 1898. Der Dogger am Espinazito-Pass. Palaeontologische Abhandlungen N.F., 8 (2), 3-69.
Volkheimer, W., Manceñido, M. y Damborenea, S. 1978a. La formación Los Patos (nov.form.), Jurásico inferior de la alta cordillera de la provincia de San Juan (República Argentina), en su localidad tipo (Río de Los Patos Sur). Revista de la Asociación Geológica Argentina, 32 (4), 300-311.

Volkheimer, W., Manceñido, M.O. und Damborenea, S.E. 1978b. Zur Biostratigraphie des Lias in der Hochkordillere von San Juan, Argentina. Münstersche Forschungen zur Geologie Paläontologie, 44/45, 205-235.

Westermann, G.E.C. and Riccardi, A.C. 1972. Middle Jurassic Ammonoid Fauna and Biochronology of the Argentine-Chilean Andes. Part I: Hildocerataceae. Palaeontographica A, 140, 1-116.

Manuscrito recibido: 20 de enero, 1995 Manuscrito aceptado: 6 de octubre, 1995 